\title{
Is adjuvant chemotherapy better than neoadjuvant chemotherapy for those with node positive disease?
}

\author{
Joseph Zabell, Badrinath Konety \\ Department of Urology, University of Minnesota, Minneapolis, MN, USA \\ Correspondence to: Joseph Zabell, MD. Department of Urology, University of Minnesota, Minneapolis, MN, USA. Email: zabe0034@umn.edu. \\ Provenance: This is an invited Editorial commissioned by Section Editor Xiao Li (Department of Urology, Jiangsu Cancer Hospital \& Jiangsu \\ Institute of Cancer Research \& Nanjing Medical University Affiliated Cancer Hospital, Nanjing, China). \\ Comment on: Cha EK, Sfakianos JP, Sukhu R, et al. Poor prognosis of bladder cancer patients with occult lymph node metastases treated with \\ neoadjuvant chemotherapy. BJU Int 2018;122:627-32.
}

Submitted Aug 03, 2018. Accepted for publication Aug 13, 2018.

doi: $10.21037 /$ tau.2018.08.21

View this article at: http://dx.doi.org/10.21037/tau.2018.08.21

The authors present a retrospective analysis of patients who have undergone radical cystectomy and been found to have pathologic lymph node metastases. Patients were stratified by their receipt of either neoadjuvant chemotherapy (NAC), or adjuvant chemotherapy (AC) with the goal of comparing outcomes between these two groups. In short, they found that 3-year recurrence free survival was markedly worse for patients in the NAC group vs. the AC group (26\% vs. $60 \%$, respectively). Patients with positive nodes after NAC had higher risk of both disease recurrence and cancer-specific mortality in univariable and multivariable analyses.

The findings are surprising as they challenge current dogma that NAC is more proven to deliver a survival benefit while the data with AC are not as definitive. These data do reaffirm findings from most studies that the benefit of NAC is only derived by patients who have a complete or a partial response to NAC. Patients with residual lymph node disease post NAC are not in that category. While the magnitude of the difference in this recurrence-free survival is striking, it is important to note the small sample size in this analysis ( 81 patients), as well as the fact that 5 patients from the cohort who received both NAC and AC were excluded from the analysis. Was survival in these patients any better? If so one could advocate for "sandwich" chemotherapy. Prior studies suggest that those patients treated with NAC and AC have similar outcomes to patients receiving AC alone (1). However, the one study examining this concept was not restricted to patients with lymph node metastases alone. It is to be pointed out that none of the patients who had residual lymph node disease received AC. It is possible that if they had received AC in addition to NAC their outcome would be much improved. When comparing data such as these, one has to be aware of the immortal time bias. Patients undergoing NAC have to be alive to undergo cystectomy and hence are subject to an "immortal" survival time from diagnosis to cystectomy. A landmark time analysis at a set point in time (e.g., 1 or $2 \mathrm{y}$ ) following radical cystectomy to assess survival would often address this concern.

An additional set of 5 patients appear to have been excluded from the $\mathrm{AC}$ cohort due to disease recurrence prior to receipt of AC. These patients were considered to have received "salvage chemotherapy" and would otherwise have represented the poorest prognosis in the AC cohort. Thus, their inclusion may have altered the overall findings and would have resulted in a decrease of the 3-year recurrence-free survival to approximately $54 \%$. While this would represent a relatively modest decrease, this highlights that subtle changes to such a small cohort can result in significant alterations to complex clinical outcomes. While the authors do appropriately address this limitation in their discussion, the inherent risk of selection bias obligates the reader to interpret these results with caution.

When considering the clinical implications of this manuscript, it is important not to misinterpret these data in such a way as to diminish the well-established role of NAC. Patients who are found to have positive nodes at cystectomy after completing NAC are a clinically distinct, chemotherapy refractory group of patients. As such, it is not terribly surprising that they ultimately fare worse than chemotherapy-naïve patients receiving $\mathrm{AC}$ and 
direct comparison of these groups to one another has the potential to yield misleading results. It is important to emphasize that NAC is associated with downsizing of primary bladder tumors (2) with even partial downstaging associated with improved outcomes (3). NAC is also associated with lower incidence of occult lymph node metastases at time of cystectomy (4). These results, in conjunction with the well-established overall survival benefits of NAC (5), have led to NAC becoming a critical component of most major guidelines for the treatment of muscle-invasive bladder cancer (6). The data associating AC with improved survival while generally positive are not as definitive and are derived from smaller underpowered randomized studies.

In the end, this work adds to a body of literature that has previously identified that non-responders to NAC have poorer survival than responders $(7,8)$. However surgical consolidation would still be important. Radical cystectomy with lymphadenectomy can improve survival post NAC even in patients with regionally extensive disease, particularly if it is restricted to the pelvis and has demonstrated response (9). Hence it should be pursued and AC considered. Identifying these patients as exceedingly high risk for recurrence aids in postsurgical patient counseling, as well as consideration for trials of adjuvant therapy with novel agents. This will be particularly important as new systemic agents and immunotherapies gain further clinical utility in the setting of urothelial carcinoma resistant to chemotherapy.

\section{Acknowledgements}

None.

\section{Footnote}

Conflicts of Interest: The authors have no conflicts of interest to declare.

Cite this article as: Zabell J, Konety B. Is adjuvant chemotherapy better than neoadjuvant chemotherapy for those with node positive disease? Transl Androl Urol 2018;7(Suppl 6):S751-S752. doi: 10.21037/tau.2018.08.21

\section{References}

1. Millikan R, Dinney C, Swanson D, et al. Integrated therapy for locally advanced bladder cancer: final report of a randomized trial of cystectomy plus adjuvant M-VAC versus cystectomy with both preoperative and postoperative M-VAC. J Clin Oncol 2001;19:4005-13.

2. Petrelli F, Coinu A, Cabiddu M, et al. Correlation of pathologic complete response with survival after neoadjuvant chemotherapy in bladder cancer treated with cystectomy: a meta-analysis. Eur Urol 2014;65:350-7.

3. Zargar H, Zargar-Shoshtari K, Lotan Y, et al. Final Pathological Stage after Neoadjuvant Chemotherapy and Radical Cystectomy for Bladder Cancer-Does pT0 Predict Better Survival than pTa/Tis/T1? J Urol 2016;195:886-93.

4. Mertens LS, Meijer RP, Meinhardt W, et al. Occult lymph node metastases in patients with carcinoma invading bladder muscle: incidence after neoadjuvant chemotherapy and cystectomy vs after cystectomy alone. BJU Int 2014;114:67-74.

5. Grossman HB, Natale RB, Tangen CM, et al. Neoadjuvant chemotherapy plus cystectomy compared with cystectomy alone for locally advanced bladder cancer. $\mathrm{N}$ Engl J Med 2003;349:859-66. Erratum in: N Engl J Med 2003;349:1880.

6. Chang SS, Bochner BH, Chou R, et al. Treatment of NonMetastatic Muscle-Invasive Bladder Cancer: AUA/ASCO/ ASTRO/SUO Guideline. J Urol 2017;198:552-9.

7. Manoharan M, Katkoori D, Kishore TA, et al. Outcome after radical cystectomy in patients with clinical $\mathrm{T} 2$ bladder cancer in whom neoadjuvant chemotherapy has failed. BJU Int 2009;104:1646-9.

8. Nieuwenhuijzen JA, Bex A, Meinhardt W, et al. Neoadjuvant methotrexate, vinblastine, doxorubicin and cisplatin for histologically proven lymph node positive bladder cancer. J Urol 2005;174:80-5.

9. Herr HW, Donat SM, Bajorin DF. Post-chemotherapy surgery in patients with unresectable or regionally metastatic bladder cancer. J Urol 2001;165:811-4. 